\title{
Epitaxial $\mathrm{Pb}$ on InAs nanowires
}

\author{
Thomas Kanne, ${ }^{1, *}$ Mikelis Marnauza, ${ }^{1}$ Dags Olsteins, ${ }^{1}$ Damon J. Carrad, ${ }^{1}$ Joachim E. Sestoft, ${ }^{1}$ Joeri de \\ Bruijckere, ${ }^{1,2}$ Lunjie Zeng, ${ }^{3}$ Erik Johnson, ${ }^{1}$ Eva Olsson, ${ }^{3}$ Kasper Grove-Rasmussen, ${ }^{1}$ and Jesper Nygård ${ }^{1, \dagger}$ \\ ${ }^{1}$ Center For Quantum Devices, Niels Bohr Institute, \\ University of Copenhagen, 2100 Copenhagen, Denmark \\ ${ }^{2}$ Kavli Institute of Nanoscience, Delft University of Technology, 2628 CJ Delft, The Netherlands \\ ${ }^{3}$ Department of Physics, Chalmers University of Technology, Gothenburg, Sweden
}

\begin{abstract}
Semiconductor-superconductor hybrids are widely used for realising complex quantum phenomena such as topological superconductivity and spins coupled to Cooper pairs. Accessing exotic regimes at high magnetic fields and increasing operating temperatures beyond the state-of-the-art requires new, epitaxially matched semiconductor-superconductor materials. The challenge is to generate favourable conditions for heterostructure formation between materials with the desired inherent properties. Here, we harness increased knowledge of metal-on-semiconductor growth to develop InAs nanowires with epitaxially matched, single crystal, atomically flat $\mathrm{Pb}$ films along the entire nanowire. These highly ordered heterostructures have a critical temperature of $7 \mathrm{~K}$ and a superconducting gap of $1.25 \mathrm{meV}$, which remains hard at $8.5 \mathrm{~T}$, thereby more than doubling the available parameter space. Additionally, InAs/Pb 'island' devices exhibit magnetic field-driven transitions from Cooper pair to single electron charging; a pre-requisite for use in topological quantum computation. Introducing semiconductor- $\mathrm{Pb}$ hybrids potentially enables access to entirely new regimes for an array of quantum systems.
\end{abstract}

The development of high-quality semiconductorsuperconductor heterostructures underlies the pursuit of new types of quantum bits based on exotic physics arising at the hybrid interface ${ }^{1-9}$. Aside from the ability to host novel two-level systems 10 14, when the underlying materials exhibit strong spin-orbit coupling, a high $g$ factor and 'hard gap' induced superconductivity 1 , they are ideally suited for supporting topological superconductivity under applied magnetic field $\frac{133|6| 14 \mid 16}{18}$. Signatures of topological superconductivity in these systems have been studied almost exclusively using InAs or InSb as the semiconductor and $\mathrm{Al}$ and $\mathrm{Nb}(\mathrm{TiN})$ as the superconductor, with the large spin-orbit coupling and $g$ factor ensuring the suitability of these semiconductors ${ }^{1}$. By contrast, there is strong interest in improving the superconductor material to broaden the scope of hybrid applications $8|19| 20$, since $\mathrm{Al}$ has a relatively low critical temperature, $T_{\mathrm{C}}$ and field $B_{\mathrm{C}} \sqrt{15}$, while the soft gap of Nb-based hybrids has thus far prevented isolated hybrid segments supporting Cooper pair charging8|18|19|21|22, a crucial requirement for topologically protected qubit schemes 23 . The main goal is therefore development of epitaxial hybrids exhibiting a large, hard gap resilient to high magnetic fields and elevated temperatures $8|19| 20$.

In this article we present hybrid nanowires utilising $\mathrm{Pb}$ as the superconductor, which possesses the highest bulk $T_{\mathrm{C}}$ and $B_{\mathrm{C}}$ of all elemental type-I superconductors, vastly extending the hybrid parameter space. We deposit the $\mathrm{Pb}$ films without breaking vacuum after InAs nanowire growth $2|19| 20 \mid 24$, and ensure carefully controlled conditions which promote strong thermodynamic driving forces towards single crystal formation. From transmission electron microscopy (TEM) we find a low energy domain match, as expected from modelling of the InAs/Pb interface, which enables the growth of an atomically flat single $\mathrm{Pb}$ crystal along the entire nanowire facet. All previous semiconductor-superconductor nanowire heterostructures exhibited axial grain boundaries and/or polycrystallinity $2|8| 19|20| 24$. The single crystal $\mathrm{Pb}$ suppresses the potential for impurity-generated discontinuity of the hybrid wavefunction 6 , which may occur in granular films $8|19| 20 \mid 24$. Further, the theoretical description used to optimise the epitaxial structures through consideration of thermodynamic driving forces and structural factors leads to increased understanding of metal/semiconductor epitaxy. The epitaxial InAs/Pb structure yields a hard induced superconducting energy gap of $\Delta \sim 1.25 \mathrm{meV}$, corresponding $T_{\mathrm{C}} \sim 7 \mathrm{~K}$, and $B_{\mathrm{C}}$ exceeding $8.5 \mathrm{~T}$, the highest reported values for epitaxial semiconductor-superconductor hybrids. In addition, In $\mathrm{As} / \mathrm{Pb}$ 'island' devices support Cooper pair charging due to the hard gap. At finite magnetic field, this evolves into single electron transport through a hybrid bound state $8|18| 19|21| 22 \mid 25$; a crucial feature in the context of topological quantum computation. The epitaxial structure and dramatic enhancement of the accessible parameter space represented by $\mathrm{InAs} / \mathrm{Pb}$ hybrids make them prime candidates for use in a wide range of semiconductor-superconductor hybrids based on nanowires $3|6| 10-12$, selective area grown networks $26 \mid 27$, and planar heterostructures $28 / 29$. Further, $\mathrm{Pb}$ possesses features that distinguish it from other elemental superconductors, such as two-band, strong-coupling superconductivity and a large spin-orbit coupling, which may lead to the emergence of new phenomena in semiconductorsuperconductor physics $1 / 9|10| 12|14| 30 \mid 31$.

\section{INAS/PB EPITAXY}

Aside from the attractive physical properties, the choice of $\mathrm{Pb}$ was based on analytical considerations of the 
a

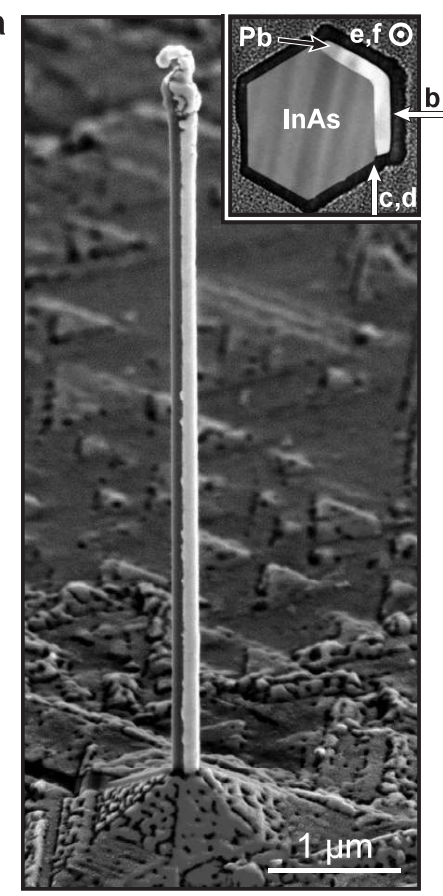

b

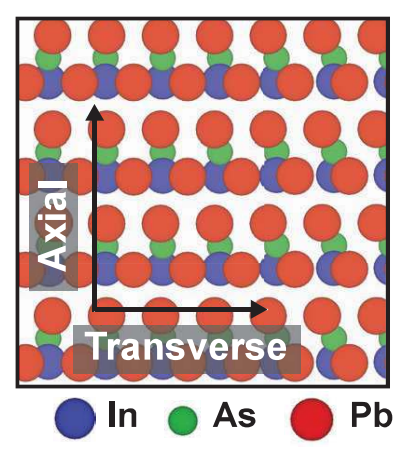

C

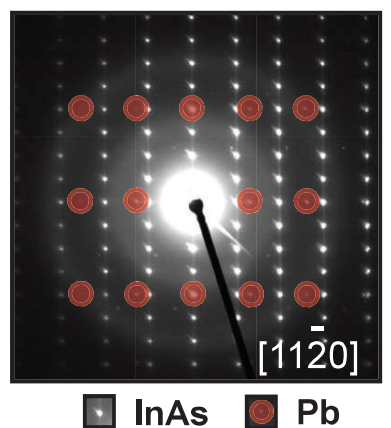

d

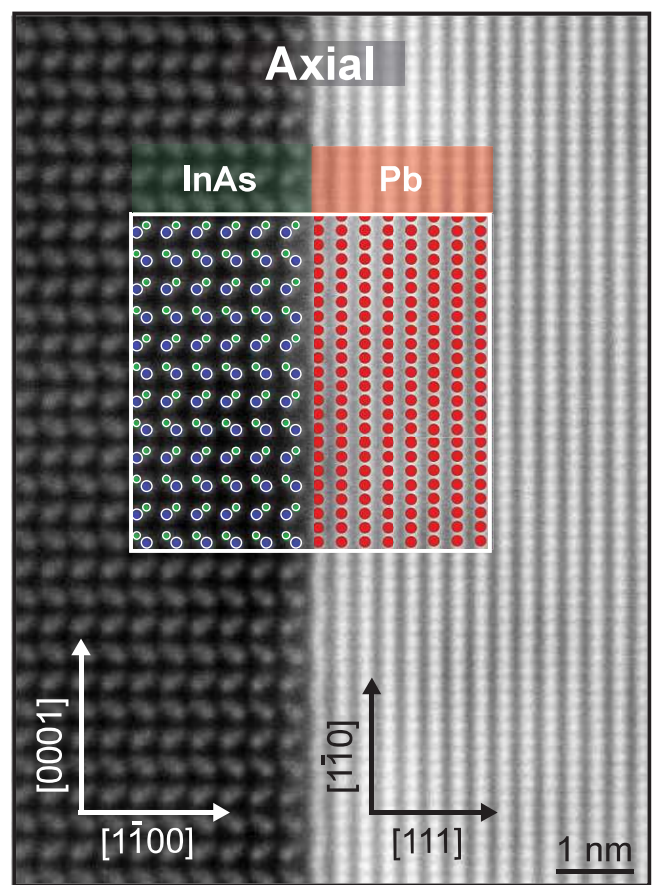

e

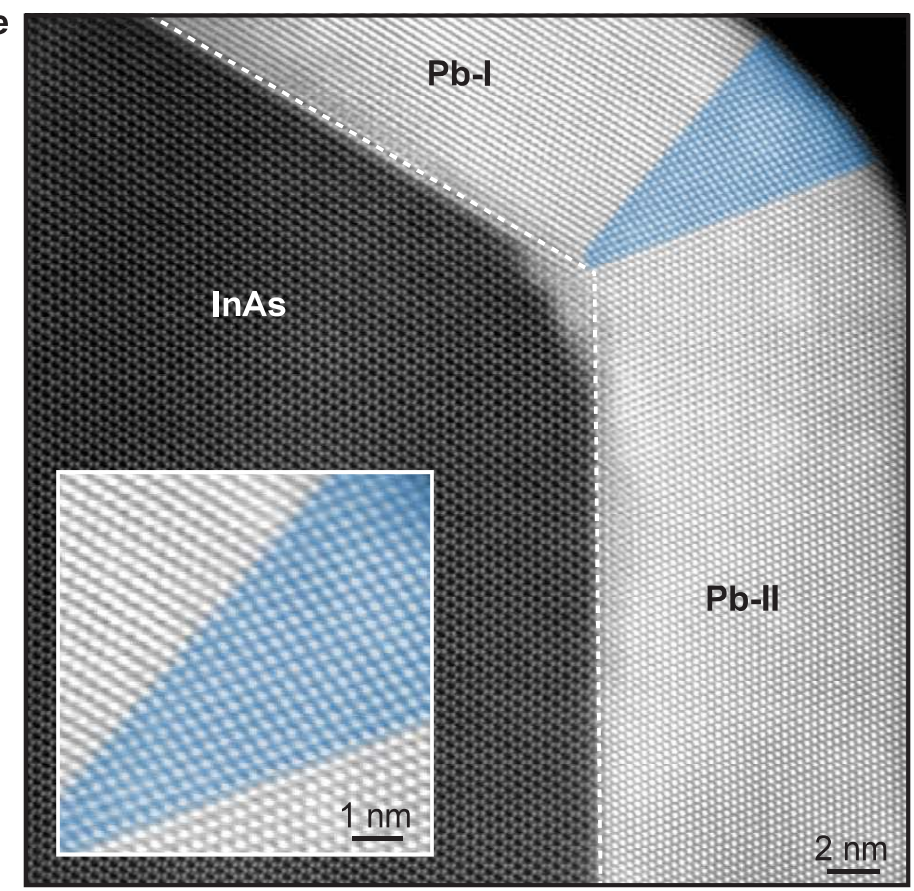

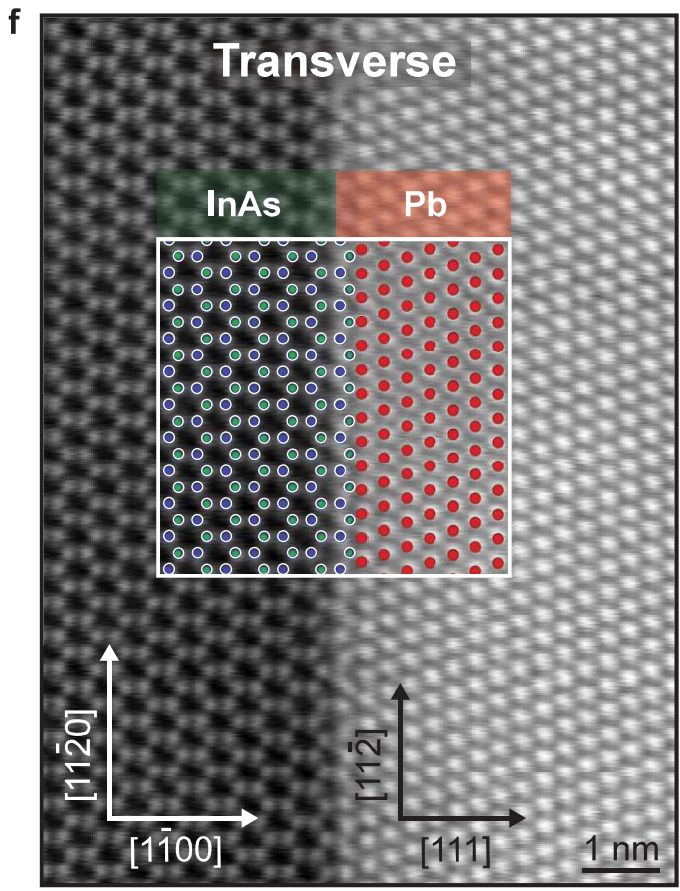

FIG. 1. InAs/Pb bi-crystal interfacial match. a, Scanning electron micrograph of an InAs nanowire with a $10 \mathrm{~nm} \mathrm{~Pb}$ thin film on two facets. Inset shows a HAADF STEM micrograph of a cross-sectioned InAs/Pb/Si heterostructure. b, Simulated relaxed bi-crystal match between InAs (blue, green) and $\mathrm{Pb}$ (red) viewed normal to the nanowire facet. c, Selected area electron diffraction pattern of an entire nanowire along the $[11 \overline{2} 0] /[11 \overline{2}]$ direction. $\mathrm{Pb}$ diffraction peaks are marked with red semi-transparent circles. d, Atomic resolution HAADF STEM micrograph of the InAs/Pb interface along the $[11 \overline{2} 0] /[11 \overline{2}]$ direction parallel to the nanowire facet. Simulated relaxed bi-crystal interfacial match overlayed and marked with a white box. e, HAADF STEM micrograph along the $[0001] /[110]$ direction of the corner between two adjacent facets, highlighted with white dotted lines. Two single $\mathrm{Pb}$ crystals are connected by a wedge shaped single crystal (false coloured blue). f, Atomic resolution HAADF STEM micrograph of the InAs/Pb interface viewed along the transverse direction as in e. Theoretically relaxed bulk bi-crystal interfacial structure superimposed on interface. 

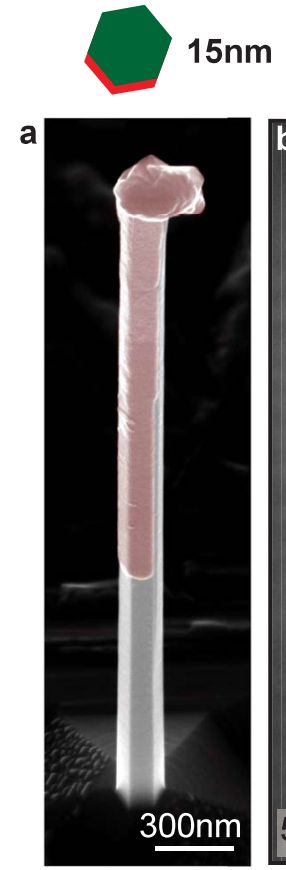

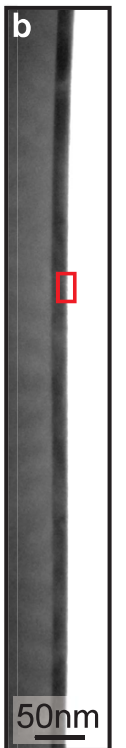

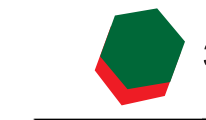

$30 \mathrm{~nm}$

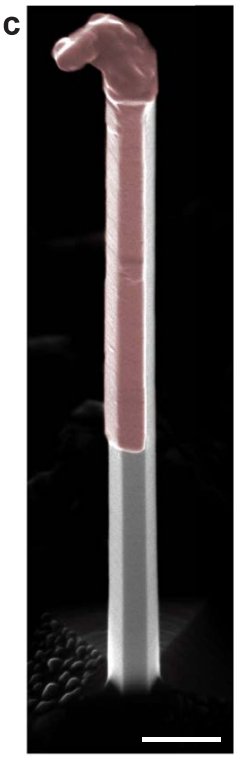

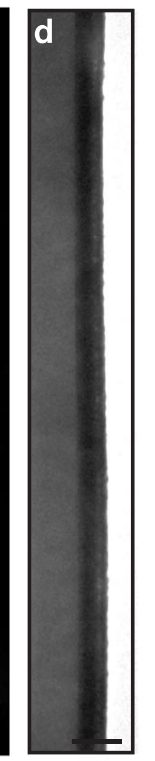

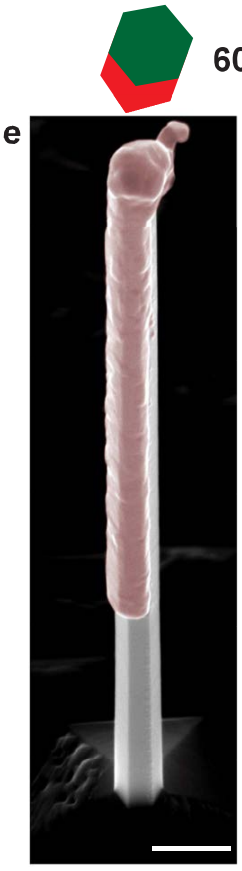

$60 \mathrm{~nm}$
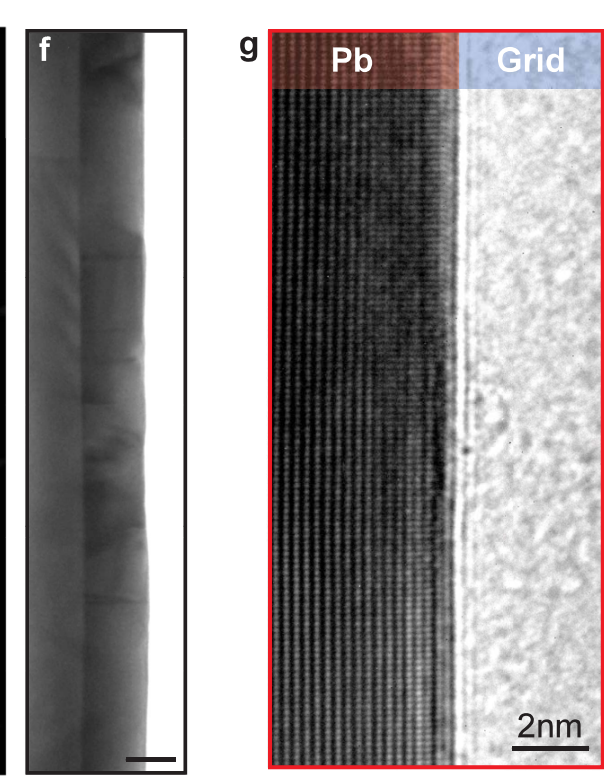

FIG. 2. Pb thickness dependent morphology. Pb grown on two facets of the InAs nanowires masked by adjacent nanowires. a,c and e show surface sensitive SEM micrographs of $15 \mathrm{~nm}, 30 \mathrm{~nm}$ and $60 \mathrm{~nm}$ in-situ deposited Pb films, respectively. The micrographs have the same scale shown in $\mathbf{a}$. $\mathbf{b}, \mathbf{d}$ and $\mathbf{f}$ present LR-TEM micrographs of same thicknesses and same scale shown in b. g HR-TEM micrograph of the terminating layer of a $15 \mathrm{~nm}$ thin film marked with a red square in $\mathbf{b}$.

central stages in metal film growth on different semiconductor nanowires. To do this, we theoretically assessed structural stability in combination with estimates of the interplay between the four contributions to the overall excess energy, which determine the thermodynamic driving forces and kinetic limiting factors. A full description is given in Supplementary Section 1 and an additional 12 elemental superconductors on InAs nanowires are theoretically studied in Supplementary Section 2. For low temperature depositions $(T<150 \mathrm{~K})$ it is energetically favourable for $\mathrm{Pb}$ to grow in a single crystallographic orientation with respect to InAs. Combined with the high grain boundary mobility of $\mathrm{Pb}$, this suggests that growth of $\mathrm{Pb}$ on InAs would result in large crystals with an epitaxial match to the nanowire. In contrast, epitaxial $\mathrm{Al}$ grows in 2 or 4 orientations on InAs depending on thickness ${ }^{2}$, and other superconductor materials employed so far feature no long-range order ${ }^{8 / 19|20| 24}$, consistent with our findings in Supplementary Section 2. These findings suggest $\mathrm{Pb}$ as the optimal elemental superconductor material to combine with InAs. Although we focus on $\mathrm{InAs} / \mathrm{Pb}$ nanowires in this article, we note that $\mathrm{Pb}$ will likely match well to other semiconductors (see Supplementary Section 3.1), and our results should translate to planar heterostructures 2829 and selective area grown networks 26|27. Further, the framework presented in Supplementary Section 1 is instructive in developing the range of semiconductor-metal epitaxial structures.

Figure 1 a shows a scanning electron microscope (SEM) micrograph of a hexagonal InAs nanowire with $\mathrm{Pb}$ deposited on two facets. The wurtzite InAs nanowires were grown along the $\{0001\}$ direction using molecular beam epitaxy (MBE), with flat $\{1 \overline{1} 00\}$ facets. The hexagonal morphology was optimised as outlined in Methods and Supplementary Section 3. After the nanowires were grown the wafer was transferred without breaking ultrahigh vacuum to a second chamber where the substrate was placed on a liquid nitrogen cooled substrate holder, $T \sim 120 \mathrm{~K}$, for several hours, before $\mathrm{Pb}$ was deposited using e-beam evaporation $\frac{19|20| 24}{2}$. The inset in Figure 1 a shows a low magnification high angle annular dark field scanning transmission electron microscope (HAADF STEM) micrograph of a cross-sectioned InAs/Pb/Si heterostructure. Figure 1 a shows the modeled relaxed hetero-epitaxial match along $\{1 \overline{1} 00\}(\operatorname{InAs}) /\{111\}(\mathrm{Pb})$ directions and the arrows indicate the axial and transverse direction. Figure 11; shows a selected area electron diffraction (SAED) pattern of an entire nanowire, oriented along the $\{11 \overline{2} 0\} /\{11 \overline{2}\}$ direction parallel to the nanowire facet. Notably, only one $\mathrm{Pb}$ orientation relative to the InAs nanowire facet is observed (red circles), confirming the theoretical hypothesis that a $\mathrm{Pb}$ thin film forms a single crystal along an entire nanowire facet.

Figure 11d shows a high resolution (HR) HAADF $\mathrm{STEM}$ micrograph of the InAs/ $\mathrm{Pb}$ interface where the orientation is similar to that of Fig. 17. The inset in Fig. $1 \mathrm{~d}$ shows how the theoretically predicted strain relaxed hetero-epitaxial match fits to the observed inter- 
face. From this, a particularly small bi-crystal interfacial domain is found along the $\{0001\} /\{1 \overline{1} 0\}$ nanowire growth direction, with two planes of $\mathrm{Pb}$ for each plane of InAs and a bulk residual mismatch of only $0.35 \%$. From HR TEM investigations of the entire nanowire length no edge-dislocations were found, indicating that the small strain needed to obtain the epitaxial domain is absorbed. Figure 1 presents a HAADF STEM micrograph of the cross-sectioned $\mathrm{InAs} / \mathrm{Pb}$ nanowire shown in Fig. 1 (inset). The two grains $(I, I I)$ form single crystals along their entire nanowire facet, and are merged by a wedge-shaped single-crystalline domain, false coloured blue. The inset in Figure 11e shows how the wedgeshaped grain accommodates two coherent grain boundaries and thus reduces the grain boundary energy between the single $I$ and $I I$ crystals. Strain in InAs and $\mathrm{Pb}$ along the transverse direction is observed by comparing the STEM micrograph and the predicted strain relaxed hetero-epitaxial match in Figure 1f. By utilizing the average [1ㅣㅣㅇㅣ plane spacing as a scale to measure the relative stress in the structure, $\mathrm{Pb}$ along the $[11 \overline{2}]$ direction was measured to be compressively strained $1.7 \%$ whereas InAs along [1120] direction was found to be tensile strained $\sim 0.7 \%$. Assuming that the InAs [1100] planes are not heavily influenced by the interface, the compressed $\mathrm{Pb}$ and expanded InAs indicate that the transverse bi-crystal interfacial match seeks a domain of two interface planes of InAs for three planes of $\mathrm{Pb}$. More detail is provided in Supplementary Section 3. The presented single crystal nature and epitaxial relation relative to the InAs nanowire facets was found for all investigated $\mathrm{Pb}$ thicknesses $(5-60 \mathrm{~nm})$ and $\mathrm{Pb}$ thus appears robust against stress driven terms such as incoherent grain boundaries.

To study the $\mathrm{Pb}$ film morphology, surface sensitive scanning electron microscopy (SEM) micrographs of InAs/Pb hybrids are shown in Figs 2a,c,e for 15, 30 and $60 \mathrm{~nm} \mathrm{~Pb}$ films, respectively. Patterning of the $\mathrm{Pb}$ coatings was implemented to enable comparative analysis of core/shell morphology and achieved by adjacent nanowires acting as shadow masks during $\mathrm{Pb}$ deposition 8/32/33. Figure 2b,d,f show bright field TEM micrographs for the different $\mathrm{Pb}$ thicknesses, and Fig. $2 \mathrm{~b}$ shows a HR TEM micrograph of the terminating planes of a $15 \mathrm{~nm}$ film of $\mathrm{Pb}$ marked with a red square in Fig. 2 $\mathrm{b}$. The $\mathrm{Pb}$ morphology is continuous and atomically flat for most of the investigated film thicknesses (10 nm - $30 \mathrm{~nm})$. However, above a critical thickness (in our case $\sim 50 \mathrm{~nm}$ ), dependent on the growth conditions, the surface becomes more rough (Fig. 2e). In Fig. 2f, bending contours and contrast changes are observed, indicative of strain fields in the $\mathrm{Pb}$ film. This may be caused by the increased strain imposed by the grain boundaries between the adjacent facets or a slight bending of the nanowires, likely due to either thermal expansion coefficient differences or axial strain 220 .

Obtaining a single crystal superconductor on a single crystal semiconductor is critical for hybrid and topolog- ical applications to ensure that each end of e.g. the topological system coincides with the geometrically defined end of the superconducting segment, rather than at an impurity ${ }^{6}$. Achieving the structures presented here required detailed understanding of how to control and promote epitaxial growth, via thermodynamic and grain growth kinetics considerations. We focus specifically on $\mathrm{Pb}$, with more general explanations provided in Supplementary Section 1-3. The aim during the initial phase of $\mathrm{Pb}$ thin film growth is to promote layer-by-layer-like growth. For island growth this is achieved by decreasing the critical cluster size and thereby increasing the initial concentration of critical clusters 34 . Experimentally, this was done by lowering the substrate temperature and increasing the material flux. After the initial clusters have been established they start to grow. In this phase, interface and strain energy may result in recrystallization of the clusters in a kinetically limited process due to the thermodynamic driving forces. In a later stage the clusters start to impinge and coalesce into a single crystal, which is now strongly dependent on the main contributions to the overall excess energy $35 \mid 36$. We find that it is especially important to have very well defined nanowire facets to decrease the interface and strain energy (Supplementary Section 1.1). The micrographs in Figs 2 a,c suggest that after a continuous film of $\mathrm{Pb}$ has grown, the growth switches to layer-by-layer mode. Here, small islands on the completed atomically flat layer act as nucleation sites for the next layer. Upon warming to room temperature and subsequent exposure to oxygen there is a chance that the $\mathrm{Pb}$ thin film dewets since it grows far from thermodynamic equilibrium 37 . We find that the film morphology is improved when the substrate remains on the cold holder for approximately 10 hours after terminating growth. Dewetting and oxidiation of $\mathrm{Pb}$ thin films may also be prevented by growing a capping layer with a lower dewetting probability.

\section{TUNNELING SPECTROSCOPY OF INAS/PB JUNCTIONS}

Having obtained the highly ordered epitaxial InAs/Pb heterostructures, we expect hard gap superconductivity with the inherited properties of the $\mathrm{Pb}$ film $\frac{15}{15}$. To characterise this we performed electron transport measurements on normal metal-superconductor (NS) tunnel junctions with the device geometry shown in Fig. 3 a. Prior to normal metal contact deposition, the $\mathrm{Pb}$ was selectively etched using $\mathrm{H}_{2} \mathrm{O}$, such that a bare InAs segment remained between the normal metal and superconductor (Methods and Supplementary Section 5). This segment acts as a tunnel barrier, tunable using side-gate voltage $V_{\mathrm{G}} \sqrt{3 \mid 15}$. Figure $3 \mathrm{~b}$ shows differential conductance, $g$, normalised to the normal state conductance, $g_{\mathrm{N}}=\left.g\right|_{V_{\mathrm{SD}}=2 \mathrm{mV}}$, as a function of source-drain bias voltage $V_{\mathrm{SD}}$ at zero magnetic field (red) and finite parallel magnetic field, $B_{\|}=7.5 \mathrm{~T}$ (blue). The strong $g$ sup- 
a
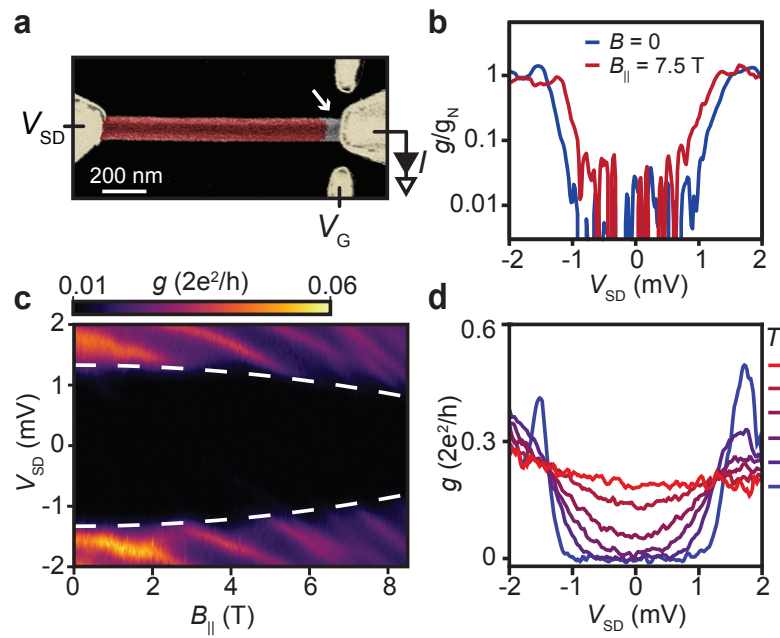

d

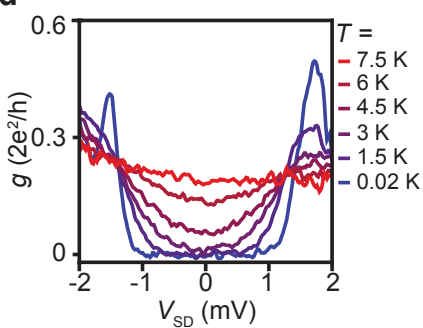

FIG. 3. Hybrid InAs/Pb tunnel spectroscopy. a, False coloured SEM micrograph showing the InAs/Pb hybrid nanowire tunnel spectroscopy device with $\mathrm{Ti} / \mathrm{Au}$ contacts and gates (yellow), $\mathrm{Pb}$ (red) and bare InAs nanowire segment (grey; also indicated by white arrow). The measurement circuit consists of source-drain bias $V_{\mathrm{SD}}$, measured current $I$, and the gate voltage $V_{\mathrm{G}}$, used to control the tunnel barrier. b, Differential conductance, $g$, normalised to the normal state conductance, $g_{\mathrm{N}}=\left.g\right|_{V_{\mathrm{SD}}=2 \mathrm{mV}}$, as a function of $V_{\mathrm{SD}}$, showing 100 -fold conductance suppression within $\pm \Delta \sim 1.25 \mathrm{meV}$ at $B=0$ (blue) and $\pm \Delta \sim 1.1 \mathrm{meV}$ for $B_{\|}=7.5 \mathrm{~T}$ (red). c, $g$ as a function of $V_{\mathrm{SD}}$ and parallel magnetic field, $B_{\|}$, showing the persistence of highly suppressed conductance until $8.5 \mathrm{~T}$. Dashed white lines serve as guide to the eye. d, $g$ as a function of $V_{\mathrm{SD}}$ recorded at increasing $T$ from 0.02 to $7.5 \mathrm{~K}$.

pression observed for $\left|V_{\mathrm{SD}}\right|<1.25 \mathrm{mV}$ reflects the superconducting energy gap, $\Delta$, in the single-particle density of states of the InAs/ $\mathrm{Pb}$ nanowire. The 100-fold suppression is similar to the hard superconducting gaps reported in III/V nanowires with epitaxial A1 $15|25| 33$, polycrystalline $\mathrm{Sn}^{8}$ and amorphous $\mathrm{Ta}{ }^{19}$, albeit here with a much larger $\Delta$. Gate voltage-dependent bias spectroscopy (Supplementary Section 4.1) shows that the gap is highly robust in $V_{\mathrm{G}}$, excluding the possibility that the features attributed to $\Delta$ in Fig. 3 are due to e.g. Coulomb blockade.

The evolution of the superconducting gap in a parallel magnetic field, $B_{\|}$, is shown in Fig. 3 c. The gap remains hard over the entire $8.5 \mathrm{~T}$ range accessible in our cryostat, highlighted by the blue trace in Fig. $3 \mathrm{~b}$. The abovegap resonances likely arise from device-specific states in the tunnel barrier. Figure $3 \mathrm{~d}$ shows the temperaturedependence of the superconducting gap. As temperature, $T$, is increased, $\Delta$ decreases and the edges broaden, up to the critical temperature $T_{\mathrm{C}} \sim 7 \mathrm{~K}$. Notably, the gap remains hard at $T=1.5 \mathrm{~K}$, and is still prominent at $T=4.5 \mathrm{~K}$. The values for $\Delta, T_{\mathrm{C}}$ and $B_{\mathrm{C}}$ of the hybrid $\mathrm{InAs} / \mathrm{Pb}$ nanowires are each more than four times larger than for state-of-the-art Al-based hybrid devices $7 / 15 / 19 \mid 25 / 33$, and exceed the metrics reported for
InSb/Sn hybrids 8 . The dramatic increase in parameter space in terms of $\Delta, T_{\mathrm{C}}$ and $B_{\mathrm{C}}$ opens the possibility of performing semiconductor-superconductor hybrid measurements at kelvin temperatures and in entirely new transport regimes 38 .

\section{COULOMB BLOCKADE SPECTROSCOPY OF INAS/PB ISLANDS}

Essential requirements for semiconductorsuperconductor heterostructures in the context of topological qubit development are for isolated hybrid segments - 'islands' - to host quantised charge in units of $2 e$, and the observation of single electron transport through bound states in finite $B_{\|}[18 \mid 21] 23|25| 39$. Figure 4 a shows an $\mathrm{InAs} / \mathrm{Pb}$ island device, with the $\mathrm{Pb}$ selectively removed at either end and contacted by $\mathrm{Ti} / \mathrm{Au}$. The side gates control the island-contact coupling and the middle gate adds charge in discrete units. Spectroscopic operation is demonstrated in Fig. 4b, with $g$ plotted versus $V_{\mathrm{SD}}$ and $V_{\mathrm{G}}$, revealing periodic Coulomb diamonds due to the sequential addition of charge in units of $2 e$ with an addition energy of $\sim 1.8 \mathrm{meV}$, which corresponds to $8 E_{\mathrm{C}}=(2 e)^{2} / C^{21}$. The regions of negative differential conductance below $\Delta$ indicate the onset of long-lifetime quasiparticle tunneling $18 \mid 21 / 22$. For $\left|V_{\mathrm{SD}}\right|>1.2 \mathrm{mV}$, the Coulomb diamond periodicity doubles in $V_{\mathrm{G}}$, since above-gap quasiparticle states support 1e-periodic transport.

The 2e-periodicity for $e\left|V_{\mathrm{SD}}\right|<\Delta$ in hard gap hybrids arises due to the odd charge states being lifted in energy by $\Delta$ compared to even charge states (Fig. 4c, top panel inset). For soft gap hybrids $\frac{40 \mid 41}{4}$ the finite density of states within the gap may enable single electron tunneling and 1e-periodicity at zero bias. The importance is that proposed topological qubit architectures rely on magnetic field induced zero energy bound states 23 , and the topologically-protected qubit operation potentially offered by these devices is obviated if soft-gap states are present. Therefore, observing both zero-field $2 e$-periodic transport and bound-state-induced $1 e$-periodic transport are highly important steps in hybrid material development.

The magnetic field dependent behaviour of our In $\mathrm{As} / \mathrm{Pb}$ island was studied using a gate voltage configuration giving a smaller addition energy of $\sim 1.2 \mathrm{meV}$ (Fig. 4c). At $B_{\|}=0$ (top panel), 2e-periodic charging is observed below $\Delta$. Increased $B_{\|}=1.1 \mathrm{~T}$ (Fig. $4 \mathrm{c}$ middle panel) causes a bound state to move towards zero energy $6 \sqrt{6 / 21 \mid 22}$, and fall below $E_{\mathrm{C}}$. Odd-state occupation is now enabled (Fig. 4c middle panel inset), with the differing even/odd charging energies producing parity-dependent, even/odd spacing of Coulomb diamonds. Further increasing $B_{\|}=1.8 \mathrm{~T}$ (lower panel) brings the bound state close to zero energy.

Figure $4 \mathrm{~d}$ presents the $B_{\|}$-dependence of $g$ versus $V_{\mathrm{G}}$ at zero bias, corresponding to the data in Fig. 4c. 
a
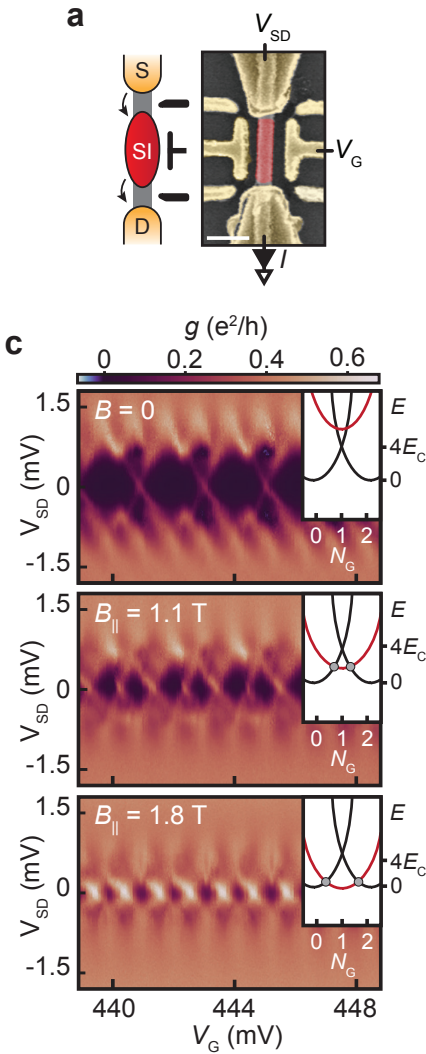

$g\left(\mathrm{e}^{2} / \mathrm{h}\right)$
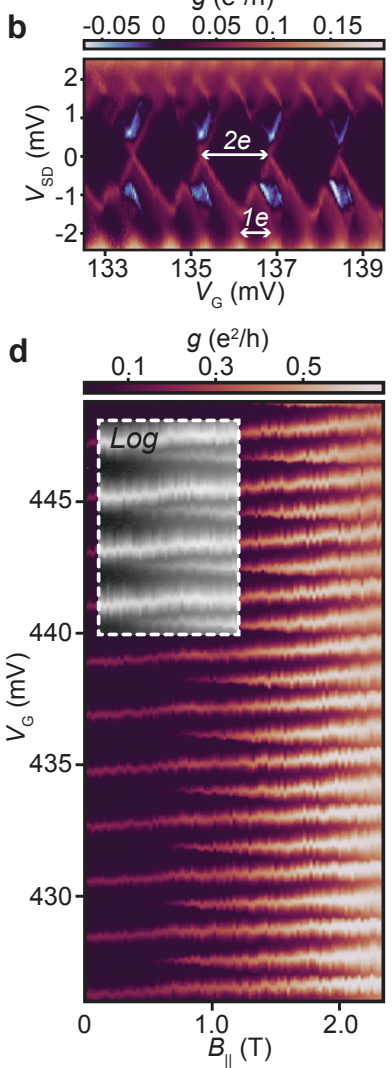

FIG. 4. Hybrid InAs/Pb island. a, Schematic of a hybrid island device. Source, drain and superconducting island are denoted S, D and SI, respectively, with the black electrostatic gates used to control single electron (or Cooper pair) tunnelling events, illustrated by the arrows. False coloured electron micrograph of a InAs/Pb island device featuring $\mathrm{Ti} / \mathrm{Au}$ contacts and gates (yellow), etched $\mathrm{Pb}$ segment (red) and narrow bare InAs segments (grey). Scale bar is $500 \mathrm{~nm}$. b, $g$ vs $V_{\mathrm{G}}$ and $V_{\mathrm{SD}}$ showing Coulomb diamonds with spacing proportional to $2 e(1 e)$ for $\left|V_{\mathrm{SD}}\right|$ less than (greater than) $\Delta / e \sim 1.2 \mathrm{mV}$, indicative of Cooper pair (single electron) charging. c, $g$ vs $V_{\mathrm{SD}}$ and $V_{\mathrm{G}}$ for $B_{\|}=0,1.1$, and $1.8 \mathrm{~T}$. Insets: Island energy, $E$, as a function of normalised gate voltage, $N_{\mathrm{G}}$. Black (red) parabolas indicate even (odd) ground state energies, $E_{\text {even }}$ and $E_{\text {odd }}$. Top panel: Cooper pair charging energy, $4 E_{\mathrm{C}}<E_{\text {odd }}$. Middle panel: $0<E_{\text {odd }}<4 E_{\mathrm{C}}$. Bottom panel: $E_{\text {even }}=E_{\text {odd }}=0$. d, Zero-bias conductance as a function of $V_{\mathrm{G}}$ and parallel magnetic field. Coulomb resonances split from a regular $2 e$ spacing $\left(4 E_{\mathrm{C}}<E_{\text {odd }}\right)$, to even/odd $\left(0<E_{\text {odd }}<4 E_{\mathrm{C}}\right)$ to a regular $1 e$ spacing $\left(E_{\text {even }}=E_{\text {odd }}=0\right)$ as a function of parallel magnetic field. Inset: Conductance plotted on logarithmic scale $\left(\log _{10}(g)\right)$ to increase visibility of the low magnetic field behavior.

The $2 e$-periodic peaks split with increasing $B_{\|}$- highlighted in the inset by the logarithmic greyscale plot — with varying even/odd spacing (see Supplementary Fig. 13). The peak intensity asymmetry between even and odd peaks can be attributed to a difference in the electron-like and hole-like components of the bound

states 821142 . These data indicate the emergence of a zero energy hybrid bound state in the In $\mathrm{As} / \mathrm{Pb}$ island, consistent with the charging model presented in the insets of Fig. 4c, and previous experiments using Al- and Sn-based hybrids $8|18| 19|21 / 22| 26$. This demonstrates that InAs/Pb hybrids meet the criteria necessary for sustaining topological superconductivity. Future work including e.g. island-length-dependent studies $\sqrt{18 \mid 22}$ or demonstrations of braiding 23 will contribute to increased understanding towards this goal.

\section{DISCUSSION}

The formation of a flat, epitaxially matched single crystal along the entire nanowire confirms the expectations from theoretical considerations of thermodynamic driving forces and structural stability of $\mathrm{Pb}$ on InAs. The framework used to investigate elemental superconductor growth on semiconductor nanowires can be extended to other metals and semiconductors, providing increased scope for development of metal/semiconductor epitaxy. In addition to the structural perfection, the epitaxial InAs/ $\mathrm{Pb}$ hybrids provide a large and hard induced superconducting gap, with corresponding high $T_{\mathrm{C}}$ and $B_{\mathrm{C}}$, offering a greatly extended experimental parameter space compared to current state-of-the-art materials. $1 / 7 / 8|15| 19|24| 33$

The observation of $2 e$-periodicity and bound-stateinduced 1e-periodic transport in an $\mathrm{InAs} / \mathrm{Pb}$ island represents an important step in the development of large $\Delta$, high $B_{\mathrm{C}}$ topological qubits $\frac{8}{\text {. Moreover, implementing } \mathrm{Pb}}$ in future devices may benefit from the strong intrinsic spin-orbit coupling of this heavy element superconductor, which has been used to induce spin-orbit coupling by proximity in, e.g., graphene ${ }^{43 / 44}$ and ferromagnets $55[46]$. In the regime of stronger spin-orbit interaction one could potentially avoid the negative aspects of the predicted metallisation effect in $\mathrm{Al}$ based devices ${ }^{47}$, where the superconductor-semiconductor coupling increases the induced gap, while decreasing the desired spin-orbit interaction. Finally, recent scanning probe experiments on Pb-based topological systems $\frac{4648}{48}$ can be complemented by exploring the strongly-coupled, two-band ${ }^{49}$ superconducting nature of $\mathrm{Pb}$ in novel hybrid devices.

\section{METHODS}

The InAs nanowires were grown in a two step growthprocess in a solid-source molecular beam epitaxy system. The nanowires were catalysed from electronbeam-lithography-defined Au particles and grown via the vapour-liquid-solid method. In the first step, InAs was grown axially for 30 minutes along the (111)B direction using $\mathrm{As}_{4}$ and a substrate thermocouple temperature $\left(T_{\text {sub }}\right)$ of $447^{\circ} \mathrm{C}$, resulting in nanowire lengths $\sim 7 \mu \mathrm{m}$. Growth was then interrupted to increase the As cracker 
temperature from $500^{\circ} \mathrm{C}$ to $800^{\circ} \mathrm{C}$, yielding $\mathrm{As}_{2}$, and the substrate temperature was lowered to $\left(T_{\text {sub }}\right)$ to $350^{\circ} \mathrm{C}$ over 20 minutes. The second growth step proceeded under these conditions, radially overgrowing the nanowires for 30 minutes.

After nanowire growth, the substrate was transferred to an attached chamber with a precooled stage without breaking ultra high vacuum (pressures $\sim 10^{-10}$ Torr). The substrate was mounted on the cold substrate stage for 3 hours before any subsequent metal deposition to ensure a stable temperature $\left(T_{\text {sub }}\right)$ of $\sim 120 \mathrm{~K}$. We note that less time on the cold substrate holder is preferred due to the possible unwanted deposition of foreign materials from the system. To enforce in-situ nanowire masking and only two facet depositions, as shown in Figs 2a,c,e, the wafer was aligned relative to the pre-defined nanowire array. $\mathrm{Pb}$ was deposited with a shallow angle and rate ( $3 \AA_{\mathrm{As}^{-1}}{ }^{-1}$ using electron beam evaporation. After the metal deposition the substrate was kept cold on the substrate holder for more than 10 hours to limit dewetting, and produced films with flatter morphology.

SEM micrographs were produced by combining signals from the surface sensitive secondary electron detector and the atomic weight sensitive backscatter electron detector since $\mathrm{Pb}$ forms a flat film which is only seen clearly when using an atomic weight sensitive technique. STEM images were acquired using an FEI Titan 80-300 TEM equipped with a probe Cs corrector and operated at $300 \mathrm{kV}$. HAADF STEM images were taken with a beam convergence semi angle of $\sim 17.5 \mathrm{mrad}$. The inner and outer collection semi angle of the HAADF detector was set to 54 and $270 \mathrm{mrad}$, respectively. Cross section specimens of the nanowires were prepared using an FEI Versa 3D focused ion beam scanning electron microscope (FIB SEM), following Pt deposition to protect the nanowires. A final polish of the TEM specimens in FIB SEM with a $2 \mathrm{kV}$ and $27 \mathrm{pA}$ ion beam was used to minimize the damage caused by the Ga ion beam. The micrograph in Fig. 1d was produced by drift correcting and summing the amplitude of 100 HR HAADF STEM micrographs with a short acquisition time. The STEM micrographs in Figs 1e,f were noise reduced by removing the Fourier spectrum background amplitude. The structural simulation of the hetero-epitaxial interface in Fig. 1 was performed using the software program Vesta ${ }^{50}$.

Devices (Figs 3 and 4) were fabricated using electron beam lithography and lift off techniques, with $\mathrm{Ti} / \mathrm{Au}(5$ $\mathrm{nm} / 250 \mathrm{~nm}$ ) thin films for contacts and gates. $\mathrm{H}_{2} \mathrm{O}$
(MilliQ with resistivity $\rho>15 \mathrm{M} \Omega$ ) was used as a selective etchant to remove $\mathrm{Pb}$ from the semiconductor surface, and $\mathrm{Ar}^{+}$milling used prior to contact deposition to remove native InAs oxides. Full details are given in the Supplementary Information. Standard voltagebiased lock-in techniques were used to measure the conductance of the devices in a dilution refrigerator with a base temperature of $25 \mathrm{mK}$. Prior to collecting the presented data on island devices (Fig. 4), the cross-coupling of the gates was measured. To collect the presented data, the gates were swept simultaneously according to the measured proportionality factors to ensure that $V_{\mathrm{G}}$ only altered island occupation and did not appreciably alter the island-lead coupling, or the chemical potential of the InAs segments.

\section{Acknowledgements}

This work was funded by the Danish National Research Foundation, European Union's Horizon 2020 research and innovation programme under grant agreement No.823717 (ESTEEM3), FETOpen grant no. 828948 (AndQC) and QuantERA project no. 127900 (SuperTOP), Villum Foundation project no. 25310, Innovation Fund Denmark's Quantum Innovation Center Qubiz, and the Carlsberg Foundation. J.d.B. acknowledges support by the Netherlands Organisation for Scientific Research (NWO/OCW), as part of the Frontiers of Nanoscience program. We thank M. Bjergfelt, A. Geresdi, T.S. Jespersen, J. Paaske, J.C.E. Saldaña and S. Vaitiekenas for useful discussions. C.B. Sørensen is gratefully acknowledged for technical assistance and support.

\section{Competing interests}

The authors declare no competing interests

\section{Supplementary Information}

Supplementary information containing extended information on metal-on-semiconductor growth, structural characterisation and transport measurements is available at https://sid.erda.dk/sharelink/eeL2CxBB6p

\footnotetext{
* thomas.kanne@nbi.ku.dk

$\dagger$ nygard@nbi.ku.dk

1 Lutchyn, R. t. et al. Majorana zero modes in superconductor-semiconductor heterostructures. Nature Reviews Materials 3, 52-68 (2018).

${ }^{2}$ Krogstrup, P. et al. Epitaxy of semiconductorsuperconductor nanowires. Nature Materials 14, 400
}

(2015).

3 Mourik, V. et al. Signatures of Majorana fermions in hybrid superconductor-semiconductor nanowire devices. Science 336, 1003-1007 (2012).

${ }^{4}$ Das, A. et al. Zero-bias peaks and splitting in an Al-InAs nanowire topological superconductor as a signature of Majorana fermions. Nature Physics 8, 887-895 (2012). 
${ }^{5}$ Deng, M. T. et al. Anomalous Zero-Bias Conductance Peak in a Nb-InSb Nanowire-Nb Hybrid Device. Nano Letters 12, 6414-6419 (2012).

${ }^{6}$ Deng, M. et al. Majorana bound state in a coupled quantum-dot hybrid-nanowire system. Science 354, 15571562 (2016).

7 Zhang, H. et al. Quantized Majorana conductance. Nature 556, 74-79 (2018).

8 Pendharkar, M. et al. Parity-preserving and magnetic field resilient superconductivity in indium antimonide nanowires with tin shells. Preprint at http://arxiv.org/abs/1912.06071 (2019).

9 Klinovaja, J. \& Loss, D. Time-reversal invariant parafermions in interacting Rashba nanowires. Phys. Rev. $B$ 90, 045118 (2014).

${ }^{10}$ Larsen, T. W. et al. Semiconductor-Nanowire-Based Superconducting Qubit. Physical Review Letters 115, 127001 (2015).

${ }^{11}$ Luthi, F. et al. Evolution of Nanowire Transmon Qubits and Their Coherence in a Magnetic Field. Physical Review Letters 120, 100502 (2018).

12 Tosi, L. et al. Spin-Orbit Splitting of Andreev States Revealed by Microwave Spectroscopy. Phys. Rev. X 9, 011010 (2019).

13 Hays, M. et al. Direct Microwave Measurement of Andreev-Bound-State Dynamics in a SemiconductorNanowire Josephson Junction. Phys. Rev. Lett. 121, 047001 (2018).

14 Prada, E. et al. From Andreev to Majorana bound states in hybrid superconductor-semiconductor nanowires. Preprint at http://arxiv.org/abs/1911.04512 (2019).

15 Chang, W. et al. Hard gap in epitaxial semiconductorsuperconductor nanowires. Nature Nanotechnology 10, 232 (2015).

16 Oreg, Y., Refael, G. \& von Oppen, F. Helical Liquids and Majorana Bound States in Quantum Wires. Physical Review Letters 105, 177002 (2010).

17 Lutchyn, R. M., Sau, J. D. \& Das Sarma, S. Majorana Fermions and a Topological Phase Transition in Semiconductor-Superconductor Heterostructures. Physical Review Letters 105, 077001 (2010).

18 Albrecht, S. M. et al. Exponential protection of zero modes in Majorana islands. Nature 531, 206 (2016).

19 Carrad, D. J. et al. Shadow Lithography for In-Situ Growth of Generic Semiconductor/Superconductor Devices. Preprint at http://arxiv.org/abs/1911.00460 (2019).

20 Bjergfelt, M. et al. Superconducting vanadium/indiumarsenide hybrid nanowires. Nanotechnology 30, 294005 (2019).

21 Shen, J. et al. Parity transitions in the superconducting ground state of hybrid InSb-Al Coulomb islands. Nature Communications 9, 4801 (2018).

${ }^{22}$ Vaitiekenas, S., Deng, M.-T., Krogstrup, P. \& Marcus, C. M. Flux-induced Majorana modes in full-shell nanowires. Preprint at http://arxiv.org/abs/1809.05513 (2018).

23 Aasen, D. et al. Milestones Toward Majorana-Based Quantum Computing. Physical Review X 6, 031016 (2016).

${ }^{24}$ Güsken, N. A. et al. MBE growth of $\mathrm{Al} / \mathrm{InAs}$ and $\mathrm{Nb} / \mathrm{InAs}$ superconducting hybrid nanowire structures. Nanoscale $\mathbf{9}$, 16735-16741 (2017)

25 Sestoft, J. E. et al. Engineering hybrid epitaxial InAsSb/Al nanowires for stronger topological protection. Physical Review Materials 2, 044202 (2018).
${ }^{26}$ Vaitiekėnas, S. et al. Selective-Area-Grown Semiconductor-Superconductor Hybrids: A Basis for Topological Networks. Physical Review Letters 121, 147701 (2018)

27 Aseev, P. et al. Selectivity Map for Molecular Beam Epitaxy of Advanced III-V Quantum Nanowire Networks. Nano Letters 19, 218-227 (2019).

${ }^{28}$ Kjaergaard, M. et al. Quantized conductance doubling and hard gap in a two-dimensional semiconductor-superconductor heterostructure. Nature Communications 7, 12841 (2016).

29 Shabani, J. et al. Two-dimensional epitaxial superconductor-semiconductor heterostructures: A platform for topological superconducting networks. Phys. Rev. B 93, 155402 (2016).

30 Sau, J. D. \& Sarma, S. D. Realizing a robust practical Majorana chain in a quantum-dot-superconductor linear array. Nature Communications 3, 964 (2012).

${ }^{31} \mathrm{Su}$, Z. et al. Andreev molecules in semiconductor nanowire double quantum dots. Nature Communications 8, 1-6 (2017).

32 Krizek, F. et al. Growth of InAs wurtzite nanocrosses from hexagonal and cubic basis. Nano Letters 17, 6090-6096 (2017).

33 Gazibegovic, S. et al. Epitaxy of advanced nanowire quantum devices. Nature 548, 434 (2017).

34 Pentcheva, R. et al. Non-Arrhenius behavior of the island density in metal heteroepitaxy: Co on $\mathrm{Cu}$ (001). Physical Review Letters 90, 076101 (2003).

35 Venables, J. \& Spiller, G. Nucleation and growth of thin films. In Surface Mobilities on Solid Materials, 341-404 (Springer, 1983).

36 Vesselinov, M. I. Crystal growth for beginners: Fundamentals of nucleation, crystal growth and epitaxy (World Scientific, 2016)

37 Thompson, C. V. Solid-state dewetting of thin films. Annual Review of Materials Research 42, 399-434 (2012).

38 Amet, F. et al. Supercurrent in the quantum Hall regime. Science 352, 966-969 (2016).

39 van Heck, B., Lutchyn, R. M. \& Glazman, L. I. Conductance of a proximitized nanowire in the Coulomb blockade regime. Phys. Rev. B 93, 235431 (2016).

40 Takei, S., Fregoso, B. M., Hui, H.-Y., Lobos, A. M. \& Das Sarma, S. Soft Superconducting Gap in Semiconductor Majorana Nanowires. Phys. Rev. Lett. 110, 186803 (2013).

${ }^{41}$ Lee, E. J. H. et al. Zero-bias anomaly in a nanowire quantum dot coupled to superconductors. Phys. Rev. Lett. 109, 186802 (2012).

42 Hansen, E. B., Danon, J. \& Flensberg, K. Probing electron-hole components of subgap states in Coulomb blockaded Majorana islands. Physical Review B 97, 041411 (2018).

43 Klimovskikh, I. I. et al. Spin-orbit coupling induced gap in graphene on $\mathrm{Pt}$ (111) with intercalated $\mathrm{Pb}$ monolayer. ACS Nano 11, 368-374 (2017).

${ }^{44}$ Calleja, F. et al. Spatial variation of a giant spin-orbit effect induces electron confinement in graphene on $\mathrm{Pb}$ islands. Nature Physics 11, 43 (2015).

45 Ruby, M., Heinrich, B. W., Peng, Y., von Oppen, F. \& Franke, K. J. Exploring a proximity-coupled Co chain on $\mathrm{Pb}(110)$ as a possible majorana platform. Nano Letters 17, 4473-4477 (2017) 
46 Nadj-Perge, S. et al. Observation of Majorana fermions in ferromagnetic atomic chains on a superconductor. Science 346, 602-607 (2014).

47 Reeg, C., Loss, D. \& Klinovaja, J. Metallization of a Rashba wire by a superconducting layer in the strongproximity regime. Phys. Rev. B 97, 165425 (2018).

48 Ménard, G. C. et al. Isolated pairs of Majorana zero modes in a disordered superconducting lead monolayer. Nature
Communications 10, 2587 (2019).

49 Ruby, M., Heinrich, B. W., Pascual, J. I. \& Franke, K. J. Experimental Demonstration of a Two-Band Superconducting State for Lead Using Scanning Tunneling Spectroscopy. Phys. Rev. Lett. 114, 157001 (2015).

50 Momma, K. \& Izumi, F. VESTA 3 for three-dimensional visualization of crystal, volumetric and morphology data. Journal of Applied Crystallography 44, 1272-1276 (2011). 